\title{
Markers of liver fibrosis and apoptosis in patients with HIV mono-infection and HIV/HCV co-infection
}

Ludmila Vīksna, Ilze Ekšteina, Monta Madelāne, Indra Zeltiņa, Angelika Krūmiṇa, Aleksandrs Koḷesovs, Raimonds Simanis

Rīga Stradiņš University, Riga, Latvia

\begin{abstract}
Introduction: Human immunodeficiency virus (HIV)/hepatits $\mathrm{C}$ virus (HCV) co-infection has a significant impact on liver-related morbidity and mortality. Among many other pathogenic mechanisms leading to accelerated hepatic disease are liver fibrosis and hepatocyte apoptosis. Hence the objective of this investigator-initiated cross-sectional study was to analyse the plasma levels of hyaluronic acid, cytokeratin-18, and cytochrome $\mathrm{c}$ in order to assess their impact on progression of liver disease in patients with HIV/HCV co-infection comparing to those with HIV mono-infection.

Material and methods: There were 80 patients with HIV infection included in the study. HCV co-infection was in $46(57.5 \%)$ patients and HIV mono-infection in 34 patients. Blood levels of hyaluronic acid were tested using a Hyaluronic Acid test Kit, cytokeratin-18 neoepitope levels using M30-Apoptose ELISA test, and cytochrome c using human Cytochrome c ELISA test.

Results: The levels of cytokeratin-18 and of hyaluronic acid were significantly higher in the group of patients with HIV/HCV co-infection. The differences in the level of cytochrome c were not significant. The analysis revealed that the main effect of HCV co-infection on the level of cytokeratin-18 is indirect. It is mediated by the level of hyaluronic acid.

Conclusions: The progression of liver disease in patients with HIV/HCV co-infection is more pronounced compared to those with HIV mono-infection. It is shown by the higher plasma levels of hyaluronic acid, which is a relevant liver fibrosis marker and hepatocyte apoptosis marker cytokeratin-18 in patients with HIV/HCV co-infection. The hyaluronic acid level is important in assessing the impact of HCV co-infection on liver apoptosis.
\end{abstract}

HIV AIDS Rev 2019; 18, 1: 33-39 DOI: https://doi.org/10.5114/hivar.2019.84198

Key words: apoptosis, hepatitis C virus, liver fibrosis, co-infection, human immunodeficiency virus (HIV).

\section{Introduction}

Due to similar routes of transmission, hepatitis $\mathrm{C}$ virus (HCV) and human immunodeficiency virus (HIV) co-infection is common in medical practice. According to

a systematic review and meta-analysis [1] there are approximately $2278400 \mathrm{HIV} / \mathrm{HCV}$ co-infections globally of which the majority, approximately 1362700 , are through drug injection. These data indicate a co-infection rate of $6.2 \%$ in HIV-infected individuals. Comparing geographic regions,

Article history:

Received: 18.06.2018

Received in revised form: 11.12.2018

Accepted: 11.12.2018

Available online: 28.03.2019
International Journal of HIV-Related Problems

HIV \& AIDS

R e v i e w 
the majority of HIV/HCV co-infected people are in Eastern Europe - approximately 607700 [1] - and sub-Saharan Africa [2]. Among countries with highest incidence of HIV infection are Estonia and Latvia. According to available data from January $1^{\text {st }} 2018$, there are 7343 known HIV-positive persons in Latvia [3]. In the time period from January 1, 2000 till December 31, 2017 there were 6135 HIV-positive patients registered in Latvia. From them 1025 (16.71\%) were co-infected with HCV [4].

$\mathrm{HIV} / \mathrm{HCV}$ co-infection has a significant impact on liverrelated morbidity and mortality due to a number of pathogenic factors. The most relevant pathogenic mechanisms leading to accelerated hepatic fibrosis among $\mathrm{HIV} / \mathrm{HCV}$ co-infected individuals are the following: direct viral effects, immune/cytokine dysregulation, altered levels of matrix metalloproteinases and fibrosis biomarkers, increased oxidative stress and hepatocyte apoptosis, HIV-associated gut depletion of CD4+ cells, and microbial translocation [5]. Other pathogenesis mechanisms include products of microbial translocation such as lipopolysaccharide (LPS) and toll-like receptor 4 (TLR4). Increased LPS blood levels are observed in patients with chronic HCV infection and in those with HIV infection. Consequently, in case of HIV/ $\mathrm{HCV}$ co-infection, progression of liver disease may be faster due to increased levels of LPS [6]. In HIV/HCV co-infected patients higher METAVIR activity scores are observed compared to patients with HCV mono-infection [7]. In patients with low levels of peripheral CD4+ T lymphocytes (200 cells/ $\mu \mathrm{l}$ ) advanced liver fibrosis and liver cirrhosis develops more frequently [8], indicating the protective role of these important immune cells.

The risk of developing advanced or end-stage liver disease in co-infected patients is definitely increased $[9,10]$. End-stage liver disease is found among the most frequent causes of death in HIV-infected, hospitalised patients [11]. Management of patients with co-infection poses a greater challenge due to the higher number of medicines used in cases of concomitant treatment of both infections [12]. Achieving sustained virologic response (SVR) is very important when treating chronic hepatitis $\mathrm{C}$ patients because in the liver stiffness stage (LSS), measured by elastometry, improvement is achieved only in those with SVR [13]. When comparing change in liver stiffness in patients with viral eradication, no significant differences were observed based on presence or absence of HIV coinfection [14].

$\mathrm{HCV}$ is also one of the leading reasons of liver transplantation worldwide. Because liver transplantation is done in more patients each year, it is important to properly manage HIV and HCV infections prior to the procedure, because it is known that outcomes are poorer in co-infected patients [15].

HIV infection leads to immune activation and increased inflammation, which may result in more significant multiorgan damage, including liver. There is evidence already from early studies that the HIV virus itself is capable of causing histological liver abnormalities [16]. Because there is no clear evidence that HIV infects hepatocytes, many studies suggest that HIV may indirectly result in hepatocellular injury [17]. There are extremely complex immune interactions in viral co-infections including differential regulatory pathways that influence virus-specific T cells [18].

One of the mechanisms by which viral infections cause pathogenic changes is through initiating the process of programmed cell death or apoptosis. Apoptosis can occur via two fundamental pathways: extrinsic and intrinsic. In the case of extrinsic pathway cell death signals are transmitted by the death receptor (DR), while the intrinsic pathway is initiated by intracellular stimuli. The intrinsic apoptosis pathway is characterised by the release of cytochrome $\mathrm{c}$ or other caspase-activating factors from the mitochondria intermembrane space into the cytoplasm [19]. As a result, apoptosome complex is formed in the cytoplasm. This complex activates caspase-9, which further activates the effector caspase cascade [20]. While in acute $\mathrm{HCV}$ infection, intensive hepatocyte apoptosis serves as a key mechanism of virus elimination, in chronic hepatitis $\mathrm{C}$ hepatocyte apoptosis is accompanied by cell proliferation and ensures persistent infection [21]. In the case of HCV infection apoptosis is not strongly correlated with the viral load but is rather immune-oriented, as shown by correlation with histology grading and infiltration of CD8-positive immune cells [22]. There is evidence that HCV core protein regulates hepatocyte apoptosis. For example, the core protein could inhibit Fas-mediated apoptosis by inhibiting the release of cytochrome $c$ and activating caspase- 9 , caspase-3, and caspase-7 [23]. Besides core protein, HCV structural proteins E1 and E2 also influence hepatocyte apoptosis; for example, E2 inhibits DR-induced apoptosis in hepatoma cells by inhibiting the release of mitochondrial cytochrome c [24].

There are increased levels of peripheral T-cell apoptosis observed in patients with chronic hepatitis $\mathrm{C}$, and the extrinsic apoptosis pathway is upregulated in both HCV monoinfection and $\mathrm{HIV} / \mathrm{HCV}$ co-infection, although this upregulation is independent of liver disease severity [25]. In the case of HIV infection, among other mechanisms T-helper cells are depleted due to apoptosis, resulting from a series of biochemical pathways [26] such as deactivation of antiapoptotic Bcl-2 by HIV enzymes. The Bcl-2-interacting protein Bid is cleaved by caspase- 8 , yielding a truncated-Bid that causes the loss of mitochondrial membrane potential and caspase- 9 cleavage, further resulting in apoptosis [27].

Hepatocyte apoptosis plays an important protective role by removing external microorganisms, including viruses. At the same time apoptosis contributes to the development of acute and chronic liver diseases, such as chronic hepatitis C. Hepatocyte apoptosis is increased in the case of HIV/ $\mathrm{HCV}$ co-infection compared to HCV infection alone, and this increase is mediated by up-regulation of death receptors 4 and 5 (DR4 and DR5), also known as tumour necrosis factor-related apoptosis-inducing ligands (TRAIL) - R1 and R2 [17].

In one study the authors concluded that HCV is a molecular-level determinant in HIV disease [28]. The authors 
found that in HIV/HCV co-infected patients T-cells were found to be destroyed at a slower rate than in patients infected HIV alone. Interestingly, in the study CD4+ and CD8+ T-cell apoptosis was lower while CD19+ B cell apoptosis was higher in $\mathrm{HIV} / \mathrm{HCV}$ co-infected patients compared with HIV mono-infection.

There are many patients worldwide with nonalcoholic fatty liver disease (NAFLD). Among other risk factors for hepatocyte apoptosis in NAFLD patients is mitochondrial dysfunction, characterised by increased plasma caspase-3 levels, which generates cytokeratin-18 fragments and soluble Fas [29].

HCV primarily attacks hepatocytes causing necrosis especially during acute infection marked by extremely high values of alanine aminotransferase (ALT) activity. In addition, hepatitis $\mathrm{C}$ infection triggers inflammation through stimulation of production of pro-inflammatory cytokines, which is associated with insulin resistance and hepatic steatosis [30] and has an association with atherosclerosis [31]. Furthermore, chronic HCV infection causes liver cirrhosis and hepatocellular carcinoma (HCC) in a relatively large proportion of infected individuals. Chronic immune activation in HIV/HCV co-infected patients may also contribute to hepatic fibrogenesis [32]. Apoptosis triggers mechanisms of cell repair in the liver - regeneration that is accompanied by inflammation. These processes lead to liver fibrogenesis. Fibrogenic activity is stimulated further by hepatic stellate cells (HSC), which engulf apoptotic bodies [33]. Hepatic stellate cells synthesise hyaluronic acid (HA), a glycosaminoglycan component of the extracellular matrix (ECM) - the basis of liver fibrosis. Hyaluronic acid is regarded as a very good (class I) biomarker of advanced liver fibrosis and cirrhosis [34]. HA has very strong negative predictive value (98-100\%), determined by its ability to rule out advanced liver fibrosis and cirrhosis [35]. More intense apoptotic processes correlate with more severe hepatic disease and more advanced [36] stage of fibrosis in patients with steatosis [37].

The aim of this investigator-initiated cross-sectional study was to analyse the plasma levels of hyaluronic acid (liver fibrosis marker), cytokeratin-18, and cytochrome c (hepatocyte apoptosis markers) to assess their impact on progression of liver disease in patients with $\mathrm{HIV} / \mathrm{HCV}$ co-infection compared to those with HIV mono-infection.

\section{Material and methods}

\section{Patients}

There were 80 patients with HIV infection included in the study. HCV co-infection was in $46(57.5 \%)$ patients while HIV mono-infection was in $34(42.5 \%)$ patients. Additionally, patients were grouped according to stage of HIV infection and presence of anti-retroviral therapy (ART). The ages of the patients ranged from 24 to 62 years, and 26 (32.5\%) were females.

\section{Data}

Data were collected from electronic patient records. The following data were registered: date of birth, gender, diagnosis (stage of HIV infection, co-infection with chronic hepatitis C), and blood serum laboratory test results (CD4+, insulin, insulin resistance [HOMA-IR], glucose, hyaluronic acid [HA], cytokeratin-18 [CK-18], cytochrome c [CYC]).

\section{Laboratory methods}

Hyaluronic acid levels (ng/ml), with normal values in the range $0-75 \mathrm{ng} / \mathrm{ml}$, were measured using a Hyaluronic Acid test Kit (Corgenix, USA). Cytokeratin-18 neoepitope levels (U/l), with normal values in the range 47.1-103.9 U/1, were quantified using the M30-Apoptose ELISA test (PEVIVA, Sweden). Cytochrome c (ng/ml), with normal value $0 \mathrm{ng} / \mathrm{ml}$, was detected using human Cytochrome c ELISA test (Bender MedSystems, Austria). HIV virus levels in plasma were measured using PCR HIV RNA test (Cobas AmpliPrep/Cobas TaqMan HIV-1 test, Roche, USA). The CD4+ lymphocyte counts were determined by flow cytometry. ALT and AST levels were detected using kinetic fermentation method. Plasma insulin was measured by Microparticle Enzyme Immunoassay (AxSym Insulin, Abbott, USA). Insulin resistance was calculated using homeostasis model assessment (HOMA-IR): fasting insulin $(\mu \mathrm{IU} / \mathrm{ml}) \times$ fasting glucose $(\mathrm{mmol} / \mathrm{l}) / 22.5$. Plasma glucose was measured by hexokinase method.

The study was initiated and monitored by the infectious diseases and dermatology department of Riga Stradiņš University. Ethical approval was provided by Rìga Stradinš University Ethics Committee on April 30, 2015 with corresponding ethical approval code No. 42/30.04.2015 and by the Medical and Biomedical Studies Ethics Committee "afonds.êtika" on May 5, 2016 with corresponding ethical approval code No. 6-A/16. The work was carried out in accordance with the Code of Ethics of the World Medical Association (Declaration of Helsinki of 1975, as revised in 2000). All included patients provided written, informed consent.

\section{Statistical analysis}

For statistical analyses IBM SPSS Statistics for Windows, Version 22.0 [38] and 'lavaan' (0.5-22) for R [39] were used. Demographic characteristics were summarised using standard descriptive methods. Data distribution was assessed using Shapiro-Wilk tests. Correlations among the markers were calculated using Spearman's rank correlation test. Comparisons between the groups were made using the Mann-Whitney $U$ test. A two-tailed $p$-value less than 0.05 was considered statistically significant. A path analysis was performed in order to assess a complex contribution of HCV to the level of the markers. Satorra-Bentler correction [40] was applied for accounting of the deviance from normal distribution of data included in the path analysis. 


\section{Results}

\section{Patients}

Altogether, 80 patients were included in the study. To study the differences in hepatocyte apoptosis markers and hyaluronic acid in their relation to $\mathrm{HIV} / \mathrm{HCV}$ co-infection, the HIV patients were divided into two groups (Table 1).

There were no statistically significant differences regarding patients' gender distribution, $\chi^{2}(1)=2.17, p=0.14$. In addition, there were no significant differences between the two groups associated with patients' age, $t(78)=1.54$, $p=0.13$, and HIV stage, $\chi^{2}(2)=1.37, p=0.51$.

\section{The markers of liver fibrosis and apoptosis}

\section{Correlations among the markers}

The relationships between plasma levels of cytokeratin-18, cytokeratin c, hyaluronic acid, CD4+ lymphocytes, glucose, insulin, and insulin resistance (HOMA-IR) were assessed with Spearman's rank correlation coefficient (Table 2). It was selected on the basis of observed non-normality (a long-tail type) of data distribution.

The analysis demonstrated a significant positive correlation between the level of cytokeratin-18 and hyaluronic acid. Hyaluronic acid correlated positively with insulin resistance and the level of insulin. Cytochrome c demonstrated positive correlations with insulin resistance, the level of insulin, and glucose. As expected, correlations among insulin resistance, the level of insulin, and glucose were positive. Correlations between the level of cytochrome $c$

Table 1. Distribution of HIV patients with or without HCV co-infection

\begin{tabular}{l|c|c}
\hline Gender & Without co-infection & With co-infection \\
\hline Male & $26(76 \%)$ & $28(61 \%)$ \\
\hline Female & $8(24 \%)$ & $18(39 \%)$ \\
\hline Total & $34(100 \%)$ & $46(100 \%)$ \\
\hline
\end{tabular}

Values shown are number of patients (\% of patients). and the level of cytokeratin 18 or hyaluronic acid were not significant. Cytokeratin-18 demonstrated no significant correlation with insulin resistance, insulin, and glucose. Hyaluronic acid did not correlate with the level of glucose. There were also no significant correlations between the level of CD4+ lymphocytes and the markers under investigation.

\section{Differences between HIV and HIV/HCV groups}

Mann-Whitney $U$-test demonstrated that the levels of cytokeratin-18 and of hyaluronic acid were significantly higher in the group of patients with HIV/HCV co-infection (Table 3). The differences in the level of cytochrome c, insulin, glucose, CD4+ lymphocytes, and insulin resistance were not significant.

The revealed differences in groups with and without HCV co-infection and correlations between the markers of liver fibrosis, apoptosis, and insulin resistance raised a question about a complex effect of HCV co-infection on the processes under investigation. The following step was performed in order to assess this effect.

\section{A complex contribution of $\mathrm{HIV} / \mathrm{HCV}$ co-infection}

In order to assess a complex contribution of $\mathrm{HIV} / \mathrm{HCV}$ co-infection to the level of hyaluronic acid, cytokeratin-18, and insulin resistance, a path model was tested (Fig. 1). The path analysis was performed using 'lavaan' for $\mathrm{R}$ [39]. A deviance of measures from a normal distribution was accounted through Satorra-Bentler correction [40]. Cytochrome $\mathrm{c}$ was excluded from the model as having no significant independent contribution to the level of insulin or insulin resistance. The level of insulin was not included in the model because of its extremely high positive correlation with insulin resistance and the relatively small number of participants.

The results of the analysis demonstrated a direct effect of HCV co-infection on the level of hyaluronic acid. In the group with $\mathrm{HIV} / \mathrm{HCV}$ co-infection, the estimated increase in the level of hyaluronic acid was $30.72 \mathrm{ng} / \mathrm{ml}$ (95\% CI from $11.19 \mathrm{ng} / \mathrm{ml}$ to $50.25 \mathrm{ng} / \mathrm{ml})$. The direct

Table 2. Spearman rank correlations among the markers in HIV patients $(N=80)$

\begin{tabular}{l|c|c|c|c|c|c}
\hline Marker & CK-18 & HA & CYC & IR & Ins & Glu \\
\hline CK-18 & 1.00 & & & & \\
\hline HA & $0.28^{*}$ & 1.00 & & & \\
\hline CYC & 0.06 & 0.05 & 1.00 & & & \\
\hline IR & 0.09 & $0.33^{* *}$ & $0.25^{*}$ & 1.00 & & \\
\hline Ins & 0.11 & $0.37^{* *}$ & $0.24^{*}$ & $0.99^{* * *}$ & 1.00 & \\
\hline Glu & -0.05 & 0.00 & $0.39^{* * *}$ & $0.50^{* * *}$ & $0.39^{* * *}$ & 1.00 \\
\hline CD4+ & 0.03 & -0.09 & -0.13 & -0.05 & -0.07 & -0.02 \\
\hline
\end{tabular}

CK-18-cytokeratin-18, HA - hyaluronic acid, CYC - cytochrome c, IR - insulin resistance, INS - insulin, Glu - glucose, CD4+-CD4+ lymphocytes ${ }^{* * *} p<0.001,{ }^{* *} p<0.01,{ }^{*} p<0.05$ 
Table 3. A comparison of the markers in HIV patients with and without HCV co-infection

\begin{tabular}{l|c|c|c}
\hline Marker & Without HCV co-infection $(n=34)$ & With HCV co-infection $(n=46)$ & $p^{\#}$ \\
\hline Hyaluronic acid $(\mathrm{ng} / \mathrm{ml})$ & $156.00 \pm 25.67$ & $235.23 \pm 45.78$ & $<0.01$ \\
\hline Cytokeratin-18 $(\mathrm{U} / \mathrm{l})$ & $21.29 \pm 3.86$ & $52.01 \pm 18.91$ & $<0.01$ \\
\hline Cytochrome c $(\mathrm{ng} / \mathrm{ml})$ & $0.33 \pm 0.29$ & $0.54 \pm 0.21$ & 0.15 \\
\hline $\begin{array}{l}\text { Insulin resistance } \\
\text { Ins } \mu \mathrm{lU} / \mathrm{ml} \times \mathrm{Glu} \mathrm{mmol} / \mathrm{l} / 22.5)\end{array}$ & $2.76 \pm 0.42$ & $2.81 \pm 0.43$ & 0.93 \\
\hline Insulin $(\mu \mathrm{lU} / \mathrm{ml})$ & $10.91 \pm 1.47$ & $11.66 \pm 1.51$ & 0.76 \\
\hline Glucose $(\mathrm{mmol} / \mathrm{l})$ & $5.44 \pm 0.15$ & $5.23 \pm 0.10$ & 0.36 \\
\hline CD4+ lymphocytes $\left(\right.$ cells $\left./ \mathrm{mm}^{3}\right)$ & $384.65 \pm 39.16$ & $342.46 \pm 34.23$ & 0.37 \\
\hline
\end{tabular}

Values are number - mean with the $95 \%$ confidence interval

"p-values for Mann-Whitney U-test

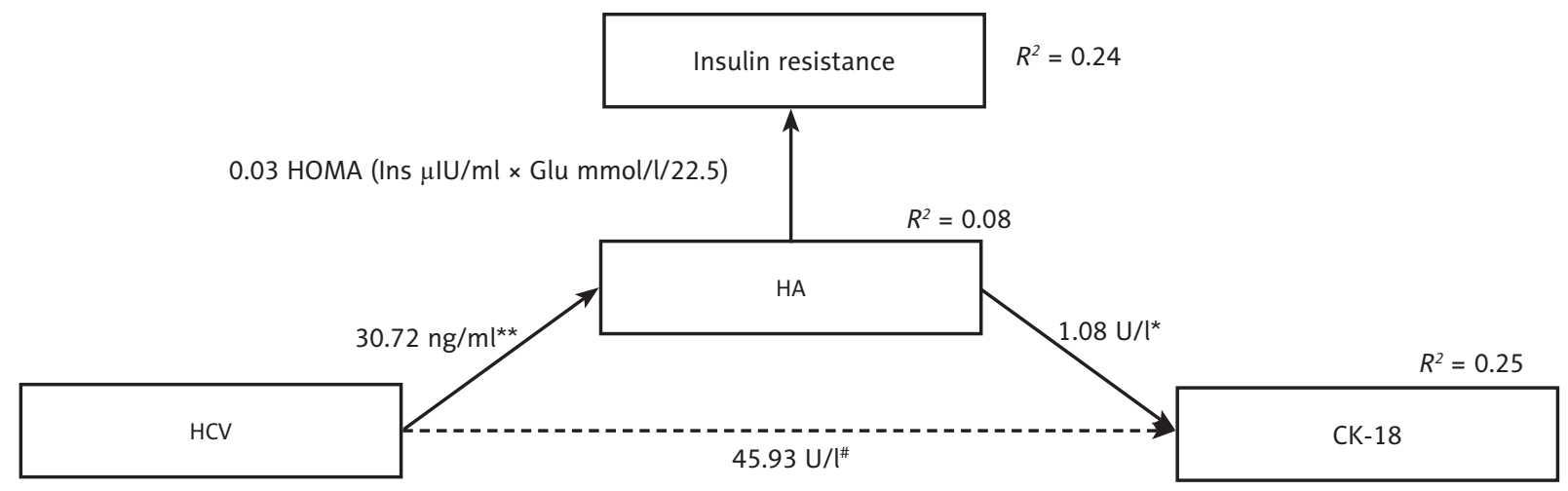

Figure 1. The path analysis on the contribution of HIV/HCV co-infection to the level of hyaluronic acid (HA), cytokeratin-18 (CK-18), and insulin resistance $\left(p<0.01^{* *}, p<0.05^{*}, p<0.10^{\#}\right)$. Dashed line shows marginal significance. Path coefficients are non-standardized estimates

effect of HIV/HCV co-infection on the level of cytokeratin-18 was only marginally significant $(p=0.08)$. The estimated change in the level of cytokeratin-18 was $45.93 \mathrm{U} / 1$ (95\% CI from $-5.63 \mathrm{U} / 1$ to $97.49 \mathrm{U} / \mathrm{l})$. The level of hyaluronic acid impacted the level of cytokeratin-18 significantly. An increase in the level of hyaluronic acid by $1.00 \mathrm{ng} / \mathrm{ml}$ was associated with an increase in the level of cytokeratin-18 by $1.08 \mathrm{U} / 1$ (95\% CI from $0.10 \mathrm{U} / 1$ to $2.07 \mathrm{U} / \mathrm{l})$. Hyaluronic acid impacted also insulin resistance. The estimated increase in insulin resistance was 0.03 HOMA (Ins $\mu \mathrm{IU} / \mathrm{ml} \times$ Glu mmol/l/22.5) and (95\% CI from 0.01 to 0.04 ).

The analysis revealed that the effects of $\mathrm{HIV} / \mathrm{HCV}$ co-infection on the level of cytokeratine- 18 and insulin resistance are indirect. They are mediated by the level of hyaluronic acid. HIV/HCV co-infection and hyaluronic acid explained $25 \%$ of variance in the level of cytokeratin- 18 and $24 \%$ of variance in insulin resistance, while HIV/HCV co-infection explained $8 \%$ of variance in the level of hyaluronic acid.

\section{Discussion}

The complex contribution of HIV/HCV co-infection analysis in this study indicates that the pathophysiology of accelerated liver fibrosis in the case of HIV infection and HIV/HCV co-infection is a complex and multifactorial process involving viral factors, the immune system, and inflammatory mediators. Advances in the understanding of fibrosis development mechanisms are very important for the planning of a liver fibrosis regulation strategy. It is well known that in the past 15-20 years progress has been made in understanding of the biology of HIV infection. However, the biochemical mechanisms of HIV-induced T-cell death, including death of uninfected CD4+ and CD8+ T cells, are not well defined.

The study confirmed a positive relationship between the markers of liver fibrosis and apoptosis - HA and CK-18. An impact of HIV/HCV co-infection on these two markers was also confirmed. As expected, the levels of HA and CK-18 were significantly higher in the group 
with HCV co-infection. In addition, the study confirmed the relationship between liver fibrosis and insulin resistance.

In all patients included in the study a significant positive correlation between the level of cytokeratin-18 and hyaluronic acid confirmed that both processes - liver fibrosis and hepatocyte and other cell apoptosis - play a significant role in the progression of liver disease. The levels of cytokeratin-18 and of hyaluronic acid were significantly higher in the group of patients with $\mathrm{HIV} / \mathrm{HCV}$ co-infection; this finding confirms that HCV presence impacts development and extent of fibrosis and apoptosis. Without a doubt the presence of HCV is the basis of the complex effect on the processes under investigation. In addition to pathogenetic effects of HIV/HCV, many other external and internal factors have a more or less prominent role in the disease progression. For example, major histocompatibility complex genes differ significantly between patients, as shown in many studies. Some patients may possess "protective gene alleles" while some possess genes that carry risk of faster disease progression.

While a direct effect of HCV on the level of hyaluronic acid is evident, the effect on the level of cytokeratin-18 is much less significant. That could be explained by different pathogenesis mechanisms in the case of HCV infection and nonalcoholic fatty liver disease with significant mitochondrial dysfunction. HIV/HCV co-infection impacts cytokeratine- 18 indirectly through the level of hyaluronic acid.

The experience of clinicians at Riga East University Hospital Latvian Centre of Infectious Diseases over many years has shown that about one third of patients with chronic HCV infection have normal serum levels of ALT. The results disclosed in the present paper suggest that the marker of apoptosis CK-18, neoepitope, should be included in the HIV infection and HIV/HCV coinfection management algorithm. Circulation HA measurements have been proposed for operative monitoring of fibrotic lesion dynamics.

The extent of liver damage cannot be assessed only by ALT level measurements. Normal plasma ALT may be misleading because there could be intensive apoptosis processes ongoing.

An important limitation of the study was associated with the sample size. It allowed testing of a simple mediation model through the path analysis. At the same time, the size of subsamples limited an assessment of the model for HIV and HIV/HCV patient groups separately. Only in rare cases is it possible to determine the time and circumstances of HIV and/or HCV infection. Consequently, patients included in the study were already in different stages of disease progression and organ damage.

Future research in the field described should be aimed at a detailed study of cell injury at a molecular level. A more complex statistical model could be developed for representing the complexity of the processes of fibrosis and apoptosis and the relationships among their markers.

\section{Conclusions}

The progression of liver disease in patients with $\mathrm{HIV} / \mathrm{HCV}$ co-infection is more pronounced comparing to those with HIV mono-infection. Besides many other parameters it is indicated by the higher plasma levels of hyaluronic acid, which is a relevant liver fibrosis marker, and hepatocyte apoptosis marker cytokeratin-18 in patients with HIV/HCV co-infection.

There is evidence for different mechanisms of impact of HIV/HCV co-infection on hyaluronic acid and CK-18. The presence of HCV impacts the level of HA directly, while the level of CK-18 is influenced through a change in the level of HA, so the stage of liver fibrosis is possibly an important factor in assessing the impact of HCV co-infection on liver apoptosis.

\section{Acknowledgments}

National research program "BIOMEDICINE", Sub-project 5.7.1. "Host organism's determinants, predisposition, and susceptibility to infections caused by RNA viruses (HIV, HCV, Tick-borne encephalitis), assessment of modulations of these determinants during the course of disease". The authors gratefully acknowledge the scientific advice of Mrs. Valentina Sondore - one of the pioneers in liver disease pathogenesis research in Latvia.

\section{Conflict of interest}

The authors declare no potential conflicts of interest with respect to the research, authorship, and/or publication of this article.

\section{References}

1. Platt L, Easterbrook P, Gower E, et al. Prevalence and burden of HCV co-infection in people living with HIV: a global systematic review and meta-analysis. Lancet Infect Dis 2016; 16: 797-808.

2. Rao VB, Johari N, du Cros P, et al. Hepatitis C seroprevalence and HIV co-infection in sub-Saharan Africa: a systematic review and meta-analysis. Lancet Infect Dis 2015; 15: 819-824.

3. Centre for Disease Prevention and Control in Latvia (in Latvian: SPKC - Slimibu profilakses un kontroles centrs). Available online: https://www.spkc.gov.lv/lv/ statistika-un-petijumi/infekcijas-slimibas/datu-vizualizacija/hivaids (accessed: 3 April 2018).

4. Centre for Disease Prevention and Control in Latvia. Letter to the authors (14.05.2018. Nr. 1-8.1.4/2611). Available on request.

5. Mastroianni CM, Lichtner M, Mascia C, et al. Molecular mechanisms of liver fibrosis in HIV/HCV coinfection. Int J Mol Sci 2014; 15: 9184-9208.

6. Sacchi P, Cima S, Corbella M, et al. Liver fibrosis, microbial translocation and immune activation markers in HIV and HCV infections and in HIV/HCV co-infection. Dig Liver Dis 2015; 47: 218-225.

7. Allory Y, Charlotte F, Benhamou Y, et al. Impact of human immunodeficiency virus infection on the histological features of chronic hepatitis C: a case-control study. The MULTIVIRC group. Hum Pathol 2000; 31: 69-74.

8. Duarte MI, Neto AN. Human immunodeficiency virus infection of the liver. In: Practical Hepatic Pathology: A Diagnostic Approach. $2^{\text {nd }}$ ed. Saxena R (ed.). Elsevier, Inc. 2018; pp. 247-264.

9. Kramer JR, Giordano TP, El-Serag HB. Effect of human immunodeficiency virus and antiretrovirals on outcomes of hepatitis C: 
a systematic review from an epidemiologic perspective. Clin Gastroenterol Hepatol 2007; 5: 1321-1328.e7.

10. Deng L, Gui X, Xiong Y, et al. End-stage liver disease: Prevalence, risk factors and clinical characteristics in a cohort of HIV-HCV coinfected Han Chinese. Clin Res Hepatol Gastroenterol 2012; 36 : 574-582.

11. Bica I, McGovern B, Dhar R, et al. Increasing mortality due to endstage liver disease in patients with human immunodeficiency virus infection. Clin Infect Dis 2001; 32: 492-497.

12. Chen JY, Feeney ER, Chung RT. HCV and HIV co-infection: mechanisms and management. Nat Rev Gastroenterol Hepatol 2014; 11: 362-371.

13. Cartón JA, Collazos J, de la Fuente B, et al. Course of liver fibrosis in HIV-hepatitis $\mathrm{C}$ virus-coinfected patients depending on the response to hepatitis C therapy. AIDS Res Hum Retroviruses 2013; 29: 215-222.

14. Singh S, Facciorusso A, Loomba R, et al. Magnitude and Kinetics of Decrease in Liver Stiffness After Antiviral Therapy in Patients With Chronic Hepatitis C: A Systematic Review and Meta-analysis. Clin Gastroenterol Hepatol 2018; 16: 27-38.e4.

15. Terrault NA, Roland ME, Schiano T, et al. Outcomes of Liver Transplantation in HCV-HIV Coinfected Recipients. Liver Transpl 2012; 18: 716-726.

16. Cao YZ, Dieterich D, Thomas PA, et al. Identification and quantitation of HIV-1 in the liver of patients with AIDS. AIDS 1992; 6: 65-70.

17. Jang JY, Shao RX, Lin W, et al. HIV infection increases HCV-induced hepatocyte apoptosis. J Hepatol 2011; 54: 612-620.

18. Cho H, Ekikuchi M, Li Y, et al. Induction of multiple immune regulatory pathways with differential impact in HCV/HIV coinfection. Front Immunol 2014; 5: 265.

19. Battaglia V, Compagnone A, Bandino A, et al. Cobalt induces oxidative stress in isolated liver mitochondria responsible for permeability transition and intrinsic apoptosis in hepatocyte primary cultures. Int J Biochem Cell Biol 2009; 41: 586-594.

20. Riedl SJ, Shi Y. Molecular mechanisms of caspase regulation during apoptosis. Nat Rev Mol Cell Biol 2004; 5: 897-907.

21. Cao L, Quan XB, Zeng WJ, et al. Mechanism of hepatocyte apoptosis. J Cell Death 2016; 9: 19-29.

22. Calabrese F, Pontisso P, Pettenazzo E, et al. Liver cell apoptosis in chronic hepatitis $\mathrm{C}$ correlates with histological but not biochemical activity or serum HCV RNA levels. Hepatology 2000; 31: 1153-1159.

23. Machida K, Tsukiyama-Kohara K, Seike E, et al. Inhibition of cytochrome $\mathrm{c}$ release in Fas-mediated signaling pathway in transgenic mice induced to express hepatitis $\mathrm{C}$ viral proteins. J Biol Chem 2001; 276: 12140-12146.

24. Lee SH, Kim YK, Kim CS, et al. E2 of hepatitis C virus inhibits apoptosis. J Immunol 2005; 175: 8226-8235.

25. Feuth T, Van Baarle D, Hoepelman AI, et al. Activation of extrinsic apoptosis pathway in HCV monoinfected and HIV-HCV coinfected patients, irrespective of liver disease severity. Apoptosis 2014; 19: 1128-1135.

26. Alimonti JB, Ball TB, Fowke KR. Mechanisms of CD4+ T lymphocyte cell death in human immunodeficiency virus infection and AIDS. J Gen Virol 2003; 84 (Pt 7): 1649-1661.

27. Yamada H, Tada-Oikawa S, Uchida A, et al. TRAIL causes cleavage of bid by caspase- 8 and loss of mitochondrial membrane potential resulting in apoptosis in BJAB cells. Biochem Biophys Res Commun 1999; 265: 130-133.

28. Laskus T, Kibler KV, Chmielewski M, et al. Effect of hepatitis C infection on HIV-induced apoptosis. PLoS One 2013; 8: e75921.

29. Tamimi TI, Elgouhari HM, Alkhouri N, et al. An apoptosis panel for nonalcoholic steatohepatitis diagnosis. J Hepatol 2011; 54: 1224-1229.

30. Zampino R, Marrone A, Restivo L, et al. Chronic HCV infection and inflammation: Clinical impact on hepatic and extra-hepatic manifestations. World J Hepatol 2013; 5: 528-540.
31. Olubamwo OO, Aregbesola AO, Miettola J, et al. Hepatitis C and risk of coronary atherosclerosis - A systematic review. Public Health 2016; 138: 12-25.

32. Rasmussen AL, Wang IM, Shuhart MC, et al. Chronic immune activation is a distinguishing feature of liver and $\mathrm{PBMC}$ gene signatures from HCV/HIV coinfected patients and may contribute to hepatic fibrogenesis. Virology 2012; 430: 43-52.

33. Canbay A, Friedman S, Gores GJ. Apoptosis: the nexus of liver injury and fibrosis. Hepatology 2004; 39: 273-278.

34. Baranova A, Lal P, Birerdinc A, Younossi ZM. Non-invasive markers for hepatic fibrosis. BMC Gastroenterol 2011; 11: 91.

35. Gressner OA, Weiskirchen R, Gressner AM. Biomarkers of liver fibrosis: clinical translation of molecular pathogenesis or based on liver dependent malfunction tests. Clin Chim Acta 2007; 381: 107-113.

36. Bantel H, Lügering A, Poremba C, et al. Caspase activation correlates with the degree of inflammatory liver injury in chronic hepatitis C virus infection. Hepatology 2001; 34: 758-767.

37. Walsh MJ, Vanags DM, Clouston AD, et al. Steatosis and liver cell apoptosis in chronic hepatitis C: a mechanism for increased liver injury. Hepatology 2004; 39: 1230-1238.

38. IBM Corp. Released 2013. IBM SPSS Statistics for Windows, Version 22.0. Armonk, NY: IBM Corp.

39. Rosseel Y. Iavaan: An R Package for Structural Equation Modeling. Journal of Statistical Software 2012; 48: 81291; DOI: 10.18637/jss. v048.i02.

40. Satorra A, Bentler PM. Corrections to test statistics and standard errors in covariance structure analysis. In: Latent Variables Analysis: Applications for Developmental Research. von Eye A, Clogg CC (eds.). Sage Publications, Inc., Thousand Oaks 1994; 399-419. 